\title{
Mitogenomics of five Olidiana leafhoppers (Hemiptera: Cicadellidae: Coelidiinae) and their phylogenetic implications
}

\author{
Xianyi Wang ${ }^{1}$, Jiajia Wang ${ }^{1}$, Ren-Huai Dai ${ }^{\text {Corresp. } 1}$ \\ 1 Institute of Entomology, Guizhou University, Guizhou Provincial Key Laboratory for Agricultural Pest Management of the Mountainous Region, Guiyang, \\ Guizhou Provincial, China \\ Corresponding Author: Ren-Huai Dai \\ Email address: rhdai@gzu.edu.cn
}

Similar morphological characteristics and limited molecular data of Olidiana resulted in their unknown phylogenetic statuses and equivocal relationships. To further understand the genus Olidiana, we sequenced and annotated five Olidiana complete mitochondrial genomes (mitogenomes). Our results show that Olidiana mitogenomes range from 15,205 bp to 15,993 bp in length and include 37 typical genes (13 protein-coding genes, 22 tRNAs, and 2 rRNAs) and a control region. Their nucleotide composition, codon usage, features of control region, and tRNA secondary structures are similar to other members of Cicadellidae. We constructed the phylogenetic tree of Cicadellidae using the maximum likelihood (ML) and Bayesian inference (BI) methods based on all valid mitogenome sequences. The most topological structure of the obtained phylogenetic tree is consistent. Our results support the monophyletic relationships among 10 subfamilies within Cicadellidae and confirm lassinae and Coelidiinae to be sister groups with high approval ratings. Interestingly, Olidiana was inferred as a paraphyletic group with strong support via both $\mathrm{ML}$ and $\mathrm{BI}$ analyses. These complete mitogenomes of five Olidiana species could be useful in further studies for species diagnosis, evolution, and phylogeny research within Cicadellidae. 
1 Mitogenomics of five Olidiana leafhoppers (Hemiptera:

2 Cicadellidae: Coelidiinae) and their phylogenetic

3 implications

Xian-Yi Wang, Jia-Jia Wang, Ren-Huai Dai

Institute of Entomology, Guizhou University; Guizhou Provincial Key Laboratory for

Agricultural Pest Management of the Mountainous Region, Guiyang 550025, P. R. China

Corresponding Author:

Ren-Huai Dai

Institute of Entomology, Guizhou University; Guizhou Provincial Key Laboratory for Agricultural Pest Management of the Mountainous Region, Guiyang 550025, P. R. China

Email address: $\underline{\text { rhdai@gzu.edu.cn }}$

\section{Abstract}

Owing to homogenous morphological characteristics and limited molecular data of Olidiana species, there is limited information on their phylogenetic status and relationships. To acquire further information on the genus Olidiana, we sequenced and annotated the complete mitochondrial genomes (mitogenomes) of five Olidiana species. The results showed that the mitogenomes ranged in length from 15,205 to 15,993 bp and included 37 typical genes (13 protein-coding genes, 22 tRNAs, and 2 rRNAs) and a control region. The nucleotide composition, codon usage, control region characteristics, and tRNA secondary structures of these mitogenomes are similar to those of other members of the Cicadellidae family. We constructed phylogenetic trees of Cicadellidae using the maximum likelihood (ML) and Bayesian inference (BI) methods based on all valid mitogenome sequences. The main topological structures of the obtained phylogenetic trees were consistent. Our results support the monophyletic relationships among 12 subfamilies within Cicadellidae and confirm Iassinae and Coelidiinae to be sister groups with high approval ratings. Interestingly, Olidiana was inferred to be a paraphyletic group, as strongly indicated by ML and BI analyses. The complete mitogenomes of the five Olidiana species could be useful to identify species, study evolution, and conduct phylogeny research within the Cicadellidae family. Subjects Entomology, Evolutionary Studies, Genomics, Molecular Biology, Taxonomy

Key words Hemiptera, Cicadellidae, paraphyletic group, mitogenome, phylogenetic analyses

\section{Introduction}

Cicadellidae is an extremely diverse family belonging to the order Hemiptera and contains an estimated 200,000 species (Oman et al., 1990; Dietrich, 2005). Since the proposal of Cicadellidae as a paraphyletic group (Cryan \& Urban, 2012; Dietrich et al., 2017), the 
40 phylogenetic relationships among its members have gained particular interest. Recently, 41 mitochondrial genomes (mitogenomes) have been widely used to infer phylogenetic relationships 42 among members of Cicadellidae. However, the primary focus has been on five subfamilies, 43 namely, Cicadellinae, Deltocephalinae, Iassinae, Idiocerinae, and Typhlocybinae, and has 44 included few representatives of Coelidiinae (Du et al., 2017a, b; Song et al., 2017, 2019; Wang et 45 al., 2017, 2019b, c; Yu et al., 2017). Coelidiinae, one of the most diverse subfamilies within 46 Cicadellidae, comprises 129 genera and more than 1,300 species (Li \& Fan, 2017; Nielson, 1980, 47 2015; Viraktamath \& Meshram, 2019; Wang et al., 2019a). Previous studies that focused on 48 morphological characteristics and molecular fragments $(H 3,28 S$, and $12 S)$ showed that 49 Coelidiinae is closely related to Cicadellinae, Evacanthinae, and Typhlocybinae (Dietrich \& 50 Deitz, 1993; Dietrich, 2001; Dietrich et al., 2010). Furthermore, Wang et al. (2019b, c, 2020a, b) reported that Coelidiinae and Iassinae form a clade at one branch and a sister group with 52 53 Macropsinae. The phylogenetic relationships among members of Coelidiinae are inconsistent based on morphological characteristics and few published mitogenomes, and additional mitogenomes may provide a better understanding of the phylogenetic relationships among the genera and species of this subfamily and among members of Cicadellidae (Dietrich et al. 2017).

Within Coelidiinae, Olidiana McKamey (2006) is a relatively large genus, with 99 reported species from the Oriental and Palearctic realms (Li \& Fan, 2017; Nielson, 1982, 2015; Viraktamath \& Meshram, 2019). Some species of Olidiana are relevant agricultural and forest pests and cause harm by directly feeding on plant sap or by indirectly spreading viral diseases (Frazier, 1975; Li \& Fan, 2017; Nielson, 1982). Olidiana exhibits morphological characteristics extremely similar to those of other Coelidiinae genera, making species distinction a challenging task. Furthermore, some species of Olidiana have been incorrectly identified, resulting in more than one synonym for the same species (Cai \& Shen, 1998; Li \& Fan, 2017; McKamey, 2000; Nielson, 1982, 2015; Walker, 1851; Xu, 2000; Zhang, 1990). In addition, the taxonomic statuses of some species of Olidiana are constantly changing, and several new genera (Singillatus, Tumidorus, and Zhangolidia) have been established by revising this genus (Li \& Fan, 2017; Nielson, 2015). Collectively, generic classification remains unsatisfactory, making it challenging to determine phylogenetic relationships. Therefore, it is necessary to utilize the mitogenomes of Olidiana species to classify and determine the genetic relationships among Coelidiinae species.

At present, 116 partial or complete mitogenomes of Cicadellidae species have been 71 deposited in GenBank. However, only three valid Coelidiinae mitogenomes [Olidiana sp., KY039119; O. ritcheriina, MK738125; and Hiatusorus fascianus (= Taharana fasciana, NC036015)] have been reported (Wang et al., 2017, 2019c). In the present study, five Olidiana species, namely, O. alata, O. longsticka, O. olbliquea, O. ritcheri, and O. tongmaiensis, representing five main groups, were identified based on their morphological characteristics (Li \& Fan, 2017; Nielson, 1982, 2015). Their mitogenomes were sequenced and annotated, and the general characteristics of the mitogenome sequences were analyzed and compared. In addition, a phylogenetic tree was constructed using Bayesian inference (BI) and maximum likelihood (ML) methods to evaluate the relationships among Cicadellidae species. 
80

81

82

83

84

85

86

87

88

89

90

91

92

93

94

95

96

97

98

99

100

101

102

103

104

105

106

107

108

109

110

111

112

113

114

115

116

117

118

119

\section{Materials and Methods}

\section{Sample collection and DNA extraction}

Details of sample collection are presented in Table S1. All specimens were preserved in absolute ethanol and stored at $-20^{\circ} \mathrm{C}$ until analysis. Genomic DNA was extracted from muscle tissues of adult males using the DNeasy ${ }^{\circledR}$ Tissue Kit (Qiagen, Germany). Total genomic DNA was eluted in $70 \mu \mathrm{L}$ of double-distilled water. The remaining extraction steps were performed according to the manufacturer's protocol. The obtained genomic DNA was stored at $-20^{\circ} \mathrm{C}$ until further analysis.

\section{Mitogenome sequencing and assembly}

Five Olidiana mitogenomes were sequenced using a next-generation sequencing platform (Illumina HiSeq 4000, Berry Genomic, Beijing, China; 6 GB raw data). Clean sequences were assembled using Geneious Primer version 2019.2.1 (Kearse et al., 2012), with O. ritcheriina (MK738125) (Wang et al., 2019c) as a reference.

\section{Mitogenome annotation and sequence analysis}

The locations of 13 protein-coding genes (PCGs) were identified using the ORF Finder tool of the National Center for Biotechnology Information and the invertebrate mitochondrial genetic code. Uncommon start and stop codons were identified by comparing our sequences with those of other Cicadellidae species. The locations and secondary structures of 22 transfer RNA (tRNA) genes were determined using tRNAscan-SE (Schattner et al., 2005) and ARWEN version 1.2 (Laslett \& Canbäck, 2008). Ribosomal RNA (rRNA) genes were identified based on the loci of adjacent tRNA genes and compared with those of other Cicadellidae species (Wang et al., 2017, 2019c). Repeat sequences within the control region were determined using the Tandem Repeats Finder tool (http://tandem.bu.edu/trf/trf.submit. options.html) (Benson et al., 1999). The annotated mitogenome sequences of the five Olidiana species have been deposited in GenBank with the accession numbers MN780581-MN780585.

Base composition and relative synonymous codon usage (RSCU) in the mitogenomes were analyzed using MEGA version 6.06 (Tamura et al., 2013). Strand asymmetry was calculated using the following formulas: AT skew $=(A-T) /(A+T)$; GC skew $=(G-C) /(G+C)($ Perna $\&$ Kocher, 1995). Intergenic spacers and overlapping regions between genes were manually counted.

\section{Sequence alignment and phylogenetic analysis}

To determine the phylogenetic relationships among members of Cicadellidae, 74 species from 12 subfamilies of Cicadellidae as well as 6 treehopper species were included, with two Cercopoidea species (Tettigades auropilosa and Cosmoscarta bispecularis) used as outgroups (Table S2). Phylogenetic analysis was performed by independently aligning the sequences of 13 PCGs and 2 rRNA genes. For each PCG sequence, terminal codons were removed before alignment using 
120 MAFFT version 7.0 in the Translator X online server (http://translatorx.co.uk/) with the L-INS-i

121

122

123

124

125

126

127

128

129

130

131

132

133

134

135

136

137

138

139

140

141

142

143

144

145

146

147

148

149

150

151

152

153

154

155

156

157

158

159 strategy (Abascal et al., 2010; Castresana et al., 2000). Each rRNA gene was individually aligned using MAFFT with the G-INS-I strategy, and poorly aligned sites were removed using Gblocks $0.91 \mathrm{~b}$ (Katoh et al., 2017). The resulting 15 alignments were concatenated using MEGA version 6.

Five datasets were concatenated for phylogenetic analysis: (1) PCGs, all codon positions of the 13 PCGs with 10,044 nucleotides; (2) PCG12, first and second codon positions of the 13 PCGs with 6,696 nucleotides; (3) AA, amino acid sequences of the 13 PCGs with 3,348 amino acids; (4) PCG12R, first and second codon positions of the 13 PCGs and 2 rRNA genes with 8,448 nucleotides; and (5) PCGR, all codon positions of the 13 PCGs and 2 rRNA genes with 11,796 nucleotides. The substitution saturation of four datasets (PCG, PCG12, PCG12R, and PCGR) was tested by plotting the number of transitions and transversions against genetic divergence using DAMBE (Xia, 2013).

A phylogenetic tree was constructed using the ML method with IQ-TREE (Nguyen et al., 2014; Zhang et al., 2019). The best-fit model was selected for each partition under corrected AIC using Partition Finder 2 (Table S3) (Lanfear et al., 2017) and evaluated using the ultrafast bootstrap approximation approach for 10,000 replicates. BI was performed using MrBayes version 3.2.6 (Suchard \& Huelsenbeck, 2012; Zhang et al., 2019). Following the partition schemes suggested by Partition Finder, all model parameters were set as unlinked across partitions. Two independent runs with four simultaneous Markov chains (one cold and three incrementally heated at $\mathrm{T}=0.2$ ) were performed for 100 million generations, with sampling every 1000 generations.

\section{Results}

\section{Mitogenomic characteristics of Olidiana species}

The complete mitogenomes of five Olidiana species, namely, O. alata (MN780581; length 15,205 bp), O. longsticka (MN780582; length 15,993 bp), O. olbliquea (MN780583; length $15,312 \mathrm{bp}$ ), O. ritcheri (MN780584; length 15,372 bp), and O. tongmaiensis (MN780585; length $15,363 \mathrm{bp}$ ), were sequenced and assembled (Table S2). Their lengths were within the ranges of complete mitogenomes reported for other Cicadellidae species $(14,805 \mathrm{bp}$ for Nephotettix cincticeps and 16,811 bp for Idioscopus laurifoliae) (Song et al., 2017; Wang et al., 2018). The mitogenomic architecture closely matched that of the inferred insect ancestral mitogenome (Crease, 1999): the newly sequenced mitogenomes had closed, circular DNA, typically comprising 37 genes (13 PCGs, 22 tRNAs, and 2 rRNAs) and a noncoding control region (Fig. 1). Of the 37 genes, most were encoded by the majority strand (J-strand) (9 PCGs and 14 tRNAs), whereas the minority strand (N-strand) encoded 14 genes (4 PCGs, 2 rRNAs, and 8 tRNAs) (Fig. 1, Table S4). However, the lengths of the 37 genes did not significantly differ between the five Olidiana species and other Cicadellidae species. The AT content of the five mitogenomes ranged from $78.0 \%$ (for $O$. alata) to 79.7\% (for O. longsticka) and displayed a positive AT skew [0.147 (for O. longsticka) to 0.195 (for $O$. tongmaiensis) ] and a negative GC

PeerJ reviewing PDF | (2020:10:53788:2:0:NEW 10 Feb 2021) 
160

161

162

163

164

165

166

167

168

169

170

171

172

173

174

175

176

177

178

179

180

181

182

183

184

185

186

187

188

189

190

191

192

193

194

195

196

197

198

199

skew [ -0.269 (for O. tongmaiensis) to -0.202 (for O. longsticka)] (Table 1). Additionally, the five Olidiana mitogenomes comprised 1-4-bp-long intergenic spacers at eight different loci, except for the trnY-COI intergenic spacer, which had 2-10-bp-long intergenic spacers. A total of 12 gene pairs were directly adjacent to each other, whereas the other gene pairs overlapped with each other, with overlap lengths of 1-4 bp, except for $\operatorname{trn} W-\operatorname{trn} C$ and $\operatorname{trn} S 2-N D 1$, which had large overlap lengths of 7-15 bp (Table S4). The gaps among the 37 genes in the mitogenomes were relatively smaller than those among genes in most reported Cicadellidae mitogenomes (Wang et al., 2018; Wang et al., 2019; Wang et al., 2020).

\section{PCGs and codon usage}

Similar to that in other reported leafhopper mitogenomes (Wang et al., 2019b, c, 2020a; Wu et al., 2016; Yang et al., 2017), in the five Olidiana mitogenomes, the lengths of the 13 PCGs ranged from $150 \mathrm{bp}(A T P 8)$ to $1,674 \mathrm{bp}$ (ND5) (Table S4). The AT content of the 13 PCGs ranged from $76.6 \%$ to $78.5 \%$. Furthermore, the PCGs displayed positive AT skew $(0.157-0.214)$ and negative GC skew ( -0.299 to -0.234 ) (Table 1). Four PCGs $(N D 4, N D 4 L, N D 5$, and $N D 1)$ were coded by the N-strand, whereas the other nine (COI, COII, COIII, ATP8, ATP6, ND2, ND3, $N D 6$, and $C Y T B$ ) were coded by the J-strand. The Olidiana mitogenomes contained similar start and stop codons, and most PCGs had the typical start codon ATN (ATA/ATT/ATG/ATC) and either TAR (TAA/TAG) or an incomplete (single T) stop codon (Table S4). The presence of incomplete stop codons is a common feature of the mitochondrial genes among other leafhoppers, particularly of ATP8, and these incomplete stop codons are most likely caused by post-transcriptional modifications during mRNA maturation (Wang et al., 2018; Wang et al., 2019; Wang et al., 2020; Yuan et al., 2019).

To understand the codon bias of the newly sequenced mitogenomes, RSCU and codon usage were determined. Codon usage was considerably similar between the five Olidiana mitogenomes and other Cicadellidae mitogenomes (Fig. 2). Among the five Olidiana mitogenomes, the most frequently used codon was UUA (for leucine; Leu). Leucine (Leu) 300-347, isoleucine (Ile) 350-404, methionine (Met) 353-375, and phenylalanine (Phe) 282-295 were the most frequently coded amino acids. However, five codons, including UCA, ACC, GUG, CCG, and GCG, were seldom used (Fig. 2). The codon usage pattern of Coelidiinae mitogenomes is highly consistent with that of previously sequenced Cicadellidae mitogenomes (Du et al., 2017; Wang et al., 2020).

\section{tRNAs and rRNAs}

Consistent with most reported leafhopper mitogenomes (Li et al., 2017b; Wang et al., 2017, 2018, 2019b, c, 2020a, b), the five Olidiana mitogenomes contained 22 tRNA genes, ranging from 57 bp (for trnC; O. longsticka) to $73 \mathrm{bp}$ (for trnK; O. tongmaiensis) in length. The AT content of the tRNA genes ranged from $78.9 \%$ to $79.4 \%$, displaying a positive AT skew $(0.111-0.145)$ and negative GC skew ( -0.150 to -0.110 ) (Table 1$)$. In addition, all tRNAs exhibited a highly conserved, canonical cloverleaf secondary structure, except trnS1 (AGN), which lacked the 
200

201

202

203

204

205

206

207

208

209

210

211

212

213

214

215

216

217

218

219

220

221

222

223

224

225

226

227

228

229

230

231

232

233

234

235

236

237

238

239

stable dihydrouridine arm commonly found in most hemipterans (Fig. 3) (Cameron, 2014; Li et al., 2017b; Wang et al., 2017, 2018, 2019b, c, 2020a, b).

Two rRNA genes ( $r r n L$ and $r r n S)$ are highly conserved in Cicadellidae mitogenomes, and each of the five Olidiana mitogenomes contained these two rRNA genes. $r r n L$ ranged from $1,176 \mathrm{bp}$ (for $O$. ritcheri) to $1,186 \mathrm{bp}$ (for $O$. longsticka) in length, whereas $r r n S$ ranged from 729 bp (in O. alata) to $788 \mathrm{bp}$ (in O. obliquea) in length (Fig. 1, Table 1). The rRNA genes of Olidiana mitogenomes displayed a positive AT skew $(0.171-0.231)$ and negative GC skew $(-0.307$ to -0.284 ) (Table 1). $r r n L$ was located between $\operatorname{trnL2}$ and $\operatorname{trn} V$, and $r r n S$ was located between $\operatorname{trn} V$ and the control region (Table S4).

\section{Control region}

The control region of Olidiana mitogenomes ranged from 1,075 bp (for O. alata) to 1,804 bp (for $O$. longsticka). The differences in length in the control region were mainly attributed to the length and number of tandem repeats $(\mathrm{R})$. All variable repeats in Olidiana mitogenomes were identified. Only a short unit (R) with two copies, both $115 \mathrm{bp}$ in length, was present in O. alata. In $O$. longsticka and $O$. olbliquea, the first repeat region (R1) was 448 and 226 bp in length, respectively, and both comprised two units. The other two repeat regions, i.e., R2 and R3, were located after R1, and they were 345 and $433 \mathrm{bp}$ (O. longsticka) and 129 and $28 \mathrm{bp}$ (O. olbliquea) in length, respectively; both comprised three copies. O. ritcheri and O. tongmaiensis comprised four types of units: R1, $2 \times 135 \mathrm{bp}$; R2, $2 \times 195 \mathrm{bp}$; R3, $3 \times 116 \mathrm{bp}$; and R4, $4 \times 78 \mathrm{bp}$ and R1, 2 $\times 281 \mathrm{bp}$; R2, $3 \times 191 \mathrm{bp}$; R3, $3 \times 81 \mathrm{bp}$; and R4, $3 \times 4 \mathrm{bp}$, respectively (Fig. 4). Similar to the long intergenic spacers in other insect species, the repeat regions in leafhoppers may be attributed to an alternative origin of mitogenome replication (Dotson \& Beard, 2001). The AT content $(84.1 \%-85.8 \%)$ of the control regions was generally higher than that of the other regions. This is in part due to damage or accumulation of mutations in the mitochondrial DNA (Martin, 1995). The control regions of the five Olidiana mitogenomes displayed a slightly positive AT skew [ranging from 0.012 (for O. ritcheri) to 0.065 (for O. longsticka)] and negative GC skew [ranging from -0.231 (for $O$. ritcheri) to -0.061 (for $O$. alata)], except the control region of $O$. longsticka, which displayed a slightly positive GC skew (0.057) (Table 1). Moreover, these control regions were compared with previously reported control region sequences; their differences were very large, and no obvious correlation or similarity was found with existing sequences.

\section{Phylogenetic relationships}

No saturation was detected among the four candidate nucleotide sequence datasets (PCGs, PCG12, PCG12R, and PCGR) prepared for ML and BI analyses (all Iss < Iss.cSym or Iss. cAsym; $P<0.05$ ) (Table S5), and the concatenated data were deemed suitable for phylogenetic analysis. Therefore, 10 phylogenetic trees were reconstructed based on five datasets (PCGs, PCG12, AA, PCG12R, and PCGR) using the BI and ML methods, and the main topological structures of the constructed phylogenetic trees were consistent (Figs. 5, 6). Our results support 
240 the view that treehoppers originated from Cicadellidae and further confirm that Cicadellidae is a 241 paraphyletic group, which is also supported by many previous studies (Du et al., 2017, 2019; Hu 242 et al., 2017; Mao et al., 2016; Song et al., 2017; Wang et al., 2018, 2020a, b; Yu et al., 2019). All 243 analyses clearly support the monophyletic relationship of the 10 subfamilies within Cicadellidae 244 and confirm that Iassinae and Coelidiinae as well as Megophthalminae and treehoppers are sister 245 groups (Figs. 5, 6, S1-S7). These results are consistent with those of previous studies (Wang et

246

247

248

249

250

251

252

253

254

255

256

257

258

259

260

261

262

263

264

265

266

267

268

269

270

271

272

273

274

275

276

277

278

279 al., 2017, 2018, 2019b, c, 2020a, b; Wu et al., 2016; Yang et al., 2017).

\section{Discussion}

The phylogenetic positions of the subfamilies Ledrinae and Deltocephalinae were different from those observed in previous studies, in which Deltocephalinae was located at the base of the Cicadellidae phylogenetic tree (Du et al., 2017; Wang et al., 2019b, c). However, in our study, the main topological structure showed that Ledrinae, instead of Deltocephalinae, was located at the base of the phylogenetic tree. This result confirms that Ledrinae is an ancient group of leafhoppers. This result is in agreement with that reported by Chen et al. (2019) and Wang et al. (2019a, b) and differs from that reported by Du et al. (2017a, b) and (Tang et al., 2020). Moreover, Tang et al. (2020) reported that there is a stable relationship between the subfamilies $[($ Coelidiinae + Iassinae $)+$ Hylicinae $]$. The results of the present study do not show such phylogenetic relationships but rather indicate that Deltocephalinae and Hylicinae are sister groups (Figs. 5, 6, S1-S7). The phylogenetic results are different from those of similar recent studies, for which there are two possible reasons: 1) we included all 13 PCGs and 2 rRNA genes in the phylogenetic analysis, whereas previous studies included 13 PCGs or selected a small number of species; 2) we included different species in the phylogenetic analysis, resulting in different conserved blocks after completion of multiple sequence alignment. In addition, phylogenetic results based on mitogenomes showed that Iassinae and Coelidiinae are sister groups; however, the phylogenetic results are different from those of Dietrich et al. (2017). Based on anchored-hybrid enrichment data, the phylogenetic results showed that the main topology was as follows: (Coelidiinae $+($ Ledrinae $+($ Hylicinae + Neobalinae $))$ ). This difference can be mainly attributed to the phylogenetic results based on different molecular data and taxa. Additional data and taxon sampling of Evacanthinae, Hylicinae, Ledrinae, Neobalinae, and Macropsinae are required to reliably determine the relationship among Cicadellidae species.

Within Coelidiinae, all phylogenetic relationships demonstrated high nodal support in both ML analyses. Interestingly, instead of clustering together, the seven Olidiana species were further divided into three groups in all analyses. The first group comprised three Olidiana species $(O$. ritcheriina $+($ Olidiana $\mathrm{sp} .+O$. ritcheri $))$, which were sister groups to $H$. fascianus (=T.fasciana, NC036015). The second group comprised three Olidiana species (O. longsticka + $(O$. olbliquea $+O$. alata) $)$, whereas the third group comprised only O. tongmaiensis. These results indicate the complex phylogenetic relationship among the species in Olidiana genus and its related genera. In particular, O. tongmaiensis, which is only distributed in the Palearctic realm, diverged from the other Olidiana species (distributed in the Oriental realm; this finding is 
280

281

282

283

284

285

286

287

288

289

290

291

292

293

294

295

296

297

298

299

300

301

302

303

304

305

306

307

308

309

310

311

312

313

314

315

316

317

318

319

consistent with their biogeographic patterns) (Li \& Fan, 2017; Viraktamath \& Meshram, 2019; Zhang, 1990). Interestingly, the seven Olidiana species could be divided into three groups based on significant differences in their morphological characteristics, which were characterized by body color, shape, and position of the processes on the aedeagus shaft. Therefore, based on complete mitogenome phylogenetic analysis and comparison of morphological characteristics, we propose Olidiana as a paraphyletic genus and suggest that it should be further examined based on the shape and position of the processes on the aedeagus shaft.

\section{Conclusions}

In this study, we sequenced and annotated the complete mitogenomes of five Olidiana species. The general genomic characteristics (gene content, gene size, gene order, base composition, PCG codon usage, and tRNA secondary structure) of the Olidiana mitogenomes were mostly consistent with those of reported Cicadellidae mitogenomes. In addition, we performed phylogenetic analyses to infer the probable relationships among the Cicadellidae subfamilies as well as to confirm the phylogenetic relationship among the Olidiana species. Our results support the presence of a monophyletic relationship among the 10 Cicadellidae subfamilies and confirm that Iassinae and Coelidiinae are sister groups with high approval ratings. Interestingly, phylogenetic analyses of the mitogenomes support our assertion that Olidiana is a paraphyletic genus, with the following topology: (O. tongmaiensis $+(O$. longsticka $+(O$. olbliquea $+O$. alata $))+(H$. fascianus $+(O$. ritcheriina $+($ Olidiana $\mathrm{sp} .+O$. ritcheri $))))$. Our findings will not only improve our understanding of the phylogenetic relationships of related insects but also contribute toward their taxonomic classification within Cicadellidae. Further studies of the combination of morphological and molecular characteristics of additional species are warranted to confirm the taxonomy of Cicadellidae.

\section{References}

Abascal F, Zardoya R, Telford MJ. 2010. TranslatorX: multiple alignment of nucleotide sequences guided by amino acid translations. Nucleic Acids Res. 38:7-13.

DOI 10.1093/nar/gkq291

Altschul SF, Madden TL, Schaffer AA, Zhang J, Zhang Z, Miller W, Lipman DJ. 1997. Gapped BLAST and PSI-BLAST: a new generation of protein database search programs. Nucleic Acids Res. 25:3389-3402. DOI 10.1093/nar/25.17.3389

Benson G. 1999. Tandem repeats finder: a program to analyze DNA sequences. Nucleic Acids Res. 27:573-580. DOI 10.1093/nar/27.2.573

Bernt M, Donath A, Juehling F, Externbrink F, Florentz C, Fritzsch G, Puetz J, Middendorf M, Stadler PF. 2013. MITOS: improved de novo metazoan mitochondrial genome annotation. Mol. Phylogen. Evol. 69:313-319. DOI 10.1016/j.ympev.2012.08.023

Cai P, Shen XC. 1998. The Fauna and Taxonomy of Insects in Henan, Vol. 2. Beijing: Agriculture Scientech Publishing House Press. 
320

321

322

323

324

325

326

327

328

329

330

331

332

333

334

335

336

337

338

339

340

341

342

343

344

345

346

347

348

349

350

351

352

353

354

355

356

357

Cameron SL. 2014. Insect mitochondrial genomics: implications for evolution and phylogeny, in: M.R. Berenbaum (Ed.). Annu. Rev. Entomol. 59:95-117.

DOI 10.1146/annurev-ento-011613-162007

Castresana J. 2000. Selection of conserved blocks from multiple alignments for their use in phylogenetic analysis. Mol. Biol. Evol. 17:540-552.

DOI 10.1093/oxfordjournals.molbev.a026334

Chen J, Wang B, Jones JR, Zheng Y, Jiang H, Jiang T, Zhang J, Zhang H. 2019. A representative of the modern leafhopper subfamily Ledrinae in mid-Cretaceous Burmese amber (Hemiptera, Cicadellidae). Cretaceous Research 95: 252-259.

DOI 10.1016/j.cretres.2018.11.022

Cryan JR, Urban JM. 2012. Higher-level phylogeny of the insect order Hemiptera: is Auchenorrhyncha really paraphyletic? Syst. Entomol. 37:7-21.

DOI 10.1111/j.1365-3113.2011.00611.x

Dietrich CH. 2010. Phylogeny of the leafhopper subfamily Evacanthinae with a review of Neotropical species and notes on related groups (Hemiptera: Membracoidea: Cicadellidae). Syst. Entomol. 29:455-487. DOI 10.1111/j.0307-6970.2004.00250.x

Dietrich CH. 2005. Keys to the families of Cicadomorpha and subfamilies and tribes of Cicadellidae (Hemiptera: Auchenorrhyncha). Fla. Entomol. 88:502-517. DOI 10.1007/0-387-31311-7_34

Dietrich CH, Allen JM, Lemmon AR, Lemmon EM, Takiya DM, Evangelista O, Walden KKO, Grady PGS, Johnson KP. 2017. Anchored hybrid enrichment-based phylogenomics of leafhoppers and treehoppers (Hemiptera: Cicadomorpha: Membracoidea). Insect Syst. Divers. 1:57-72. DOI 10.1093/isd/ixx003

Dietrich CH, Deitz LL. 1993. Superfamicaly Membracoidea (Homoptera: Auchenorrhyncha). II. Cladistic analysis and conclusions. Syst. Entomol. 18:297-311.

Dietrich CH, Rakitov RA, Holmes JL, Black WC. 2001. Phylogeny of the major lineages of Membracoidea (Insecta: Hemiptera: Cicadomorpha) based on $28 S$ rDNA sequences. Mol. Biol. Evol. 18:293-305. DOI 10.1006/mpev.2000.0873

Dotson EM, Beard CB. 2001. Sequence and organization of the mitochondrial genome of the Chagas disease vector, Triatoma dimidiata, Insect. Mol. Biol. 10:205-215. DOI 10.1046/j.1365-2583.2001.00258.x

Du Y, Dai W, Dietrich CH. 2017a. Mitochondrial genomic variation and phylogenetic relationships of three groups in the genus Scaphoideus (Hemiptera: Cicadellidae: Deltocephalinae). Sci. Rep. 7:14197. DOI 10.1038/s41598-017-17145-z

Du YM, Dietrich CH, Dai W. 2019. Complete mitochondrial genome of Macrosteles quadrimaculatus (Matsumura) (Hemiptera: Cicadellidae: Deltocephalinae) with a shared tRNA rearrangement and its phylogenetic implications. Int. J. Biol. Macromol. 122:10271034. DOI 10.1016/j.ijbiomac.2018.09.049 
358

359

360

361

362

363

364

365

366

367

368

369

370

371

372

373

374

375

376

377

378

379

380

381

382

383

384

385

386

387

388

389

390

391

392

393

394

395

396

397

Du Y, Zhang C, Dietrich CH, Zhang Y, Dai W. 2017b. Characterization of the complete mitochondrial genomes of Maiestas dorsalis and Japananus hyalinus (Hemiptera: Cicadellidae) and comparison with other Membracoidea. Sci. Rep. 7:14197. DOI 10.1038/s41598-017-14703-3

Frazier NW. 1975. Possible transmission of strawberry pallidosis by the leafhopper Coelidia olitoria. Plant Dis Report. 59:40-41.

Hu K, Yuan F, Dietrich CH, Yuan XQ. 2019. Structural features and phylogenetic implications of four new mitogenomes of Centrotinae (Hemiptera: Membracidae). Int. J. Biol. Macromol. 139:1018-1027. DOI 10.1016/j.ijbiomac.2019.08.064

Katoh K, Rozewicki J, Yamada KD. 2019. MAFFT online service: multiple sequence alignment, interactive sequence choice and visualization. Brief. Bioinform. 20:1160-1166. DOI $10.1093 / \mathrm{bib} / \mathrm{bbx} 108$

Kearse M, Moir R, Wilson A, Stones HS, Cheung M, Sturrock S, Buxton S, Cooper A, Markowitz S, Duran C. 2012. Geneious basic: an integrated and extendable desktop software platform for the organization and analysis of sequence data. Bioinformatics 28:1647-1649. DOI 10.1093/bioinformatics/bts199

Lanfear R, Frandsen PB, Wright AM, Senfeld T, Calcott B. 2017. Partitionfinder 2: new methods for selecting partitioned models of evolution for molecular and morphological phylogenetic analyses. Mol. Biol. Evol. 34:772-773. DOI 10.1093/molbev/msw260

Laslett D, Canbäck B. 2008. ARWEN: a program to detect tRNA genes in metazoan mitochondrial nucleotide sequences. Bioinformatics 24:172-175.

DOI 10.1093/bioinformatics/btm573

Li H, Leavengood JM, Chapman EG, Burkhardt D, Song F, Jiang P, Liu JP, Zhou XG, Cai WZ. 2017b. Mitochondrial phylogenomics of Hemiptera reveals adaptive innovations driving the diversification of true bugs. Roy. Soc. B-Biol. Sci. 284:20171223. DOI 10.1098/rspb.2017.1223

Li ZZ, Fan ZH. 2017. Coelidiinae (Hemiptera: Cicadellidae) from China. Guiyang: Guizhou Science and Technology Publishing House Press.

Mao M, Yang X, Bennett G. 2016. The complete mitochondrial genome of Entylia carinata (Hemiptera: Membracidae). Mitochondrial DNA Part B 1:662-663. DOI 10.1080/23802359.2016.1219629

Martin AP. 1995. Metabolic rate and directional nucleotide substitution in animal mitochondrial DNA. Mol. Biol. Evol, 12:1124-1131. DOI 10.1006/jhev.1995.1071

McKamey SH. 2006. Further new genus-group names in the Cicadellidae (Hemiptera). Proc. Entomol. Soc. Wash. 108:502-510. DOI 10.1016/j.pestbp.2005.11.009

Nguyen LT, Schmidt HA, von Haeseler A, Minh BQ. 2015. IQ-TREE: a fast and effective stochastic algorithm for estimating maximum-likelihood phylogenies. Mol. Biol. Evol. 32:268-274. DOI 10.1093/molbev/msu300

Nielson MW. 1980. A revision of the subfamily Coelidiinae (Homoptera: Cicadellidae) IV. Tribe Coelidiini. Pac. Insects Momograph 38:1-318. DOI 10.1093/besa/26.4.478 
398

399

400

401

402

403

404

405

406

407

408

409

410

411

412

413

414

415

416

417

418

419

420

421

422

423

424

425

426

427

428

429

430

431

432

433

434

435

436

437

438

Nielson MW. 2015. A revision of the tribe Coelidiini of the Oriental, Palearctic and Australian biogeographical regions (Hemiptera: Cicadellidae: Coelidiinae). Insecta Mundi 0410:1-202.

Oman PW., Knight WJ, Nielson MW. 1990. Leafhoppers (Cicadellidae): A Bibliography, Generic Checklist and Index to the World Literature. CAB International Institute of Entomology, Wallingford, United Kingdom, 368 pp.

Perna NT, Kocher TD. 1995. Patterns of nucleotide composition at fourfold degenerate sites of animal mitochondrial genomes. J. Mol. Evol. 41:353-358.

Schattner P, Brooks AN, Lowe TM. 2005. The tRNAscan-SE, snoscan and snoGPS web servers for the detection of tRNAs and snoRNAs. Nucleic. Acids. Res. 33:686-689. DOI 10.1093/nar/gki366

Song N. Cai WZ, Li H. 2017. Deep-level phylogeny of Cicadomorpha inferred from mitochondrial genomes sequenced by NGS. Sci. Rep. 7:1-11.

DOI 10.1038/s41598-017-11132-0

Song N, Zhang H, Zhao T. 2019. Insights into the phylogeny of Hemiptera from increased mitogenomic taxon sampling. Mol. Phylogen. Evol. 137:236-249.

DOI 10.1016/j.ympev.2019.05.009

Suchard MA, Huelsenbeck JP. 2012. MrBayes 3.2: efficient bayesian phylogenetic inference and model choice across a large model space. Syst. Biol. 61:539-542.

DOI 10.2307/41515220/

Tamura K, Stecher G, Peterson D, Filipski A, Kumar S. 2013. MEGA6: molecular evolutionary genetics analysis version 6.0. Mol. Biol. Evol. 30:2725-2729. DOI 10.1093/molbev/mst197

Tang J, Huang WJ, Zhang YL. 2020. The Complete Mitochondrial Genome of Four Hylicinae (Hemiptera: Cicadellidae): Structural Features and Phylogenetic Implications. Insects 11(12). DOI 10.3390/insects 11120869

Viraktamath CA, Meshram NM. 2019. Leafhopper tribe Coelidiini (Hemiptera: Cicadellidae: Coelidiinae) of the Indian subcontinent. Zootaxa 4653:1-91. DOI 10.11646/zootaxa.4653.1

Walker F. 1851. List of the specimens of homopterous insects in the collection of the British Museum. Order of the Trustees, London. 637-907.

Wang JJ, Li DF, Li H, Yang MF, Dai RH. 2019b. Structural and phylogenetic implications of the complete mitochondrial genome of Ledra auditura. Sci. Rep. 9:1-11. DOI 10.1038/s41598-019-52337-9

Wang JJ, Li H, Dai RH. 2017. Complete mitochondrial genome of Taharana fasciana (Insecta, Hemiptera: Cicadellidae) and comparison with other Cicadellidae insects. Genetica 145:593602. DOI 10.1007/s10709-017-9984-8

Wang JJ, Wu YF, Dai RH, Yang MF. 2020a. Comparative mitogenomes of six species in the subfamily Iassinae (Hemiptera: Cicadellidae) and phylogenetic analysis. Int. J. Biol. Macromol. 149:1294-1303. DOI 10.1016/j.ijbiomac.2020.01.270

Wang JJ, Wu YF, Yang M F, Dai RH. 2020b. The phylogenetic implications of the mitochondrial genomes of macropsis notata and oncopsis nigrofasciata. Front. Genet. 11:443. DOI 10.3389/fgene. 2020.00443

PeerJ reviewing PDF | (2020:10:53788:2:0:NEW 10 Feb 2021) 
439

440

441

442

443

444

445

446

447

448

449

450

451

452

453

454

455

456

457

458

459

460

461

462

463

464

465

466

467

468
Wang JJ, Yang MF, Dai RH, Wang XY. 2018. Characterization and phylogenetic implications of the complete mitochondrial genome of Idiocerinae (Hemiptera: Cicadellidae). Int. J. Biol. Macromol. 120:2366-2372. DOI 10.1016/j.ijbiomac.2018.08.191

Wang XY, Fan ZH, Li ZZ, Dai RH. 2019a. Key to genera of Chinese Coelidiinae leafhoppers, with description a new species of the leafhopper genus Baseprocessa (Hemiptera: Auchenorrhyncha: Cicadellidae). Zootaxa 4701:454-460. DOI 10.11646/zootaxa.4701.5

Wang XY, Wang JJ, Fan ZH, Dai RH. 2019c. Complete mitogenome of Olidiana ritcheriina (Hemiptera: Cicadellidae) and phylogeny of Cicadellidae. PeerJ 7:e8072. DOI $10.7717 /$ peerj.8072

Wu YF, Dai RH, Zhan HP, Qu L. 2016. Complete mitochondrial genome of Drabescoides nuchalis (Hemiptera: Cicadellidae). Mitochondrial DNA A 27:3626-3627. DOI 10.3109/19401736.2015.1079827

Xia X. 2013. DAMBE5: a comprehensive software package for data analysis in molecular biology and evolution. Mol. Biol. Evol. 30:1720-1728. DOI 10.1093/molbev/mst064

Xu GL. 2000. Three new species of Lodiana (Homoptera: Cicadellidae) from China. Entomlogia Sinica 7:218-222.

Yang XS, Mao M, Bennett G. 2017. The complete mitochondrial genome of Macrosteles quadrilineatus (Hemiptera: Cicadellidae). Mitochondrial DNA Part B 2:173-175. DOI 10.1080/23802359.2017.1303347

Yu PF, Wang MX, Cui L, Chen XX, Han BY. 2017. The complete mitochondrial genome of Tambocerus sp. (Hemiptera: Cicadellidae). Mitochondrial DNA Part A 28:133-134. DOI 10.3109/19401736.2015.1111357

Zhang D, Gao F, Jakovlic I, Zhou H, Zhang J, Li WX, Wang GT. 2019. PhyloSuite: an integrated and scalable desktop platform for streamlined molecular sequence data management and evolutionary phylogenetics studies. Mol. Ecol. Resour. 20:348-355. DOI 10.1111/1755-0998.13096

Zhang YL. 1990. A taxonomic study of Chinese Cicadellidae (Homoptera). Yangling: Tianze Publishing House Press. 


\section{Figure 1}

Figure 1. Mitochondrial genome map of five representative Olidiana species.

(A) Mitochondrial genome map of Olidiana alata. (B) Mitochondrial genome map of Olidiana longsticka. (C) Mitochondrial genome map of Olidiana olbliquea. (D) Mitochondrial genome map of Olidiana ritcheri. (E) Mitochondrial genome map of Olidiana tongmaiensis. (F) Dorsal view of five representative Olidiana species.
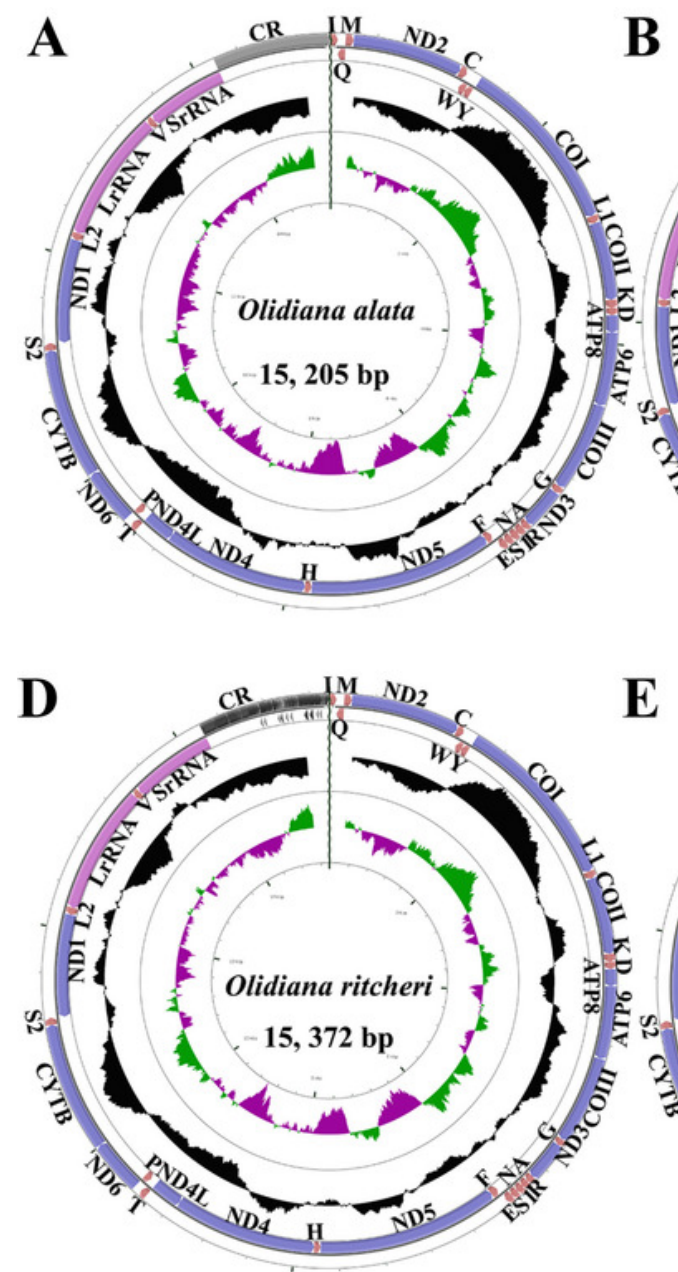

B

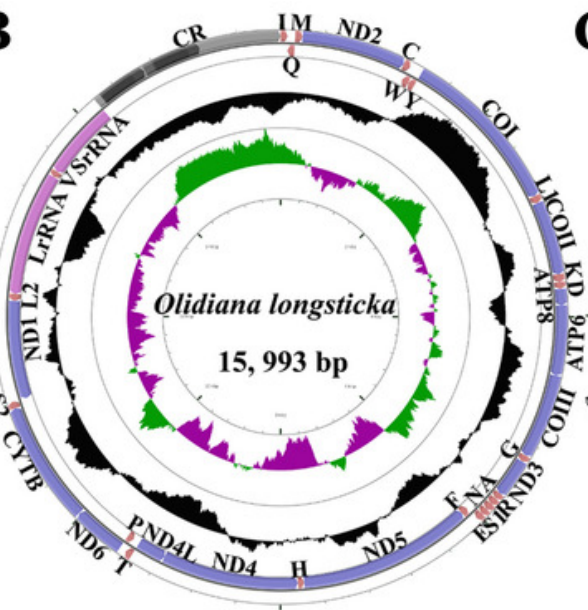

$\mathbf{E}$

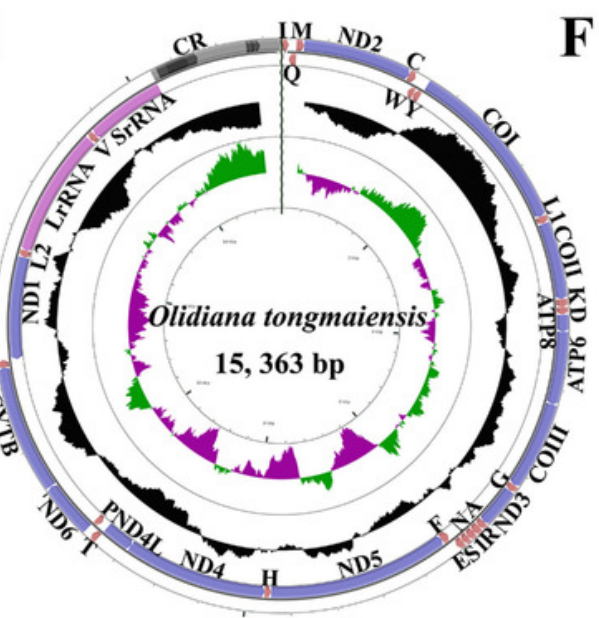

C

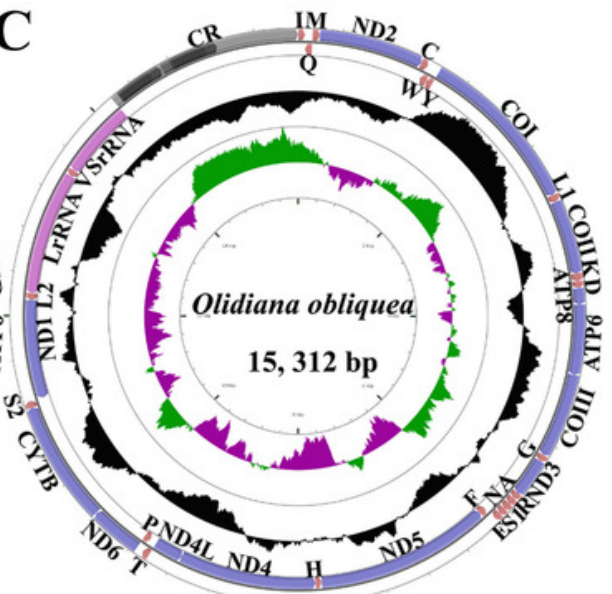

$\mathbf{F}$

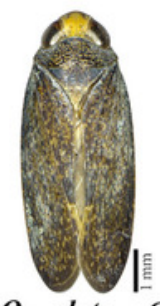

O. alata

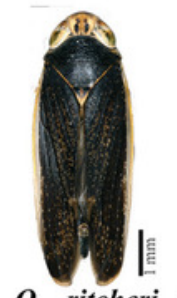

$O$. ritcheri $O$. tongmaiensis 


\section{Figure 2}

Figure 2. Relative synonymous codon usage and number of codons used in Olidiana mitogenomes.

(A) Relative synonymous codon usage in the O. alata PCGs of the mitogenome. (B) Number of codon used in O. alata PCGs of the mitogenome. (C) Relative synonymous codon usage in the O. longsticka PCGs of the mitogenome. (D) Number of codon used in O. longsticka PCGs of the mitogenome.(E) Relative synonymous codon usage in the $O$. olbliquea PCGs of the mitogenome. (F) Number of codon usage in O. olbliquea PCGs of the mitogenome. (G) Relative synonymous codon usage in the $O$. ritcheri PCGs of the mitogenome. $(\mathrm{H})$ Number of codon used in O. ritcheri PCGs of the mitogenome. (I) Relative synonymous codon usage in the $O$. tongmaiensis PCGs of the mitogenome. (J) Number of codon used in $O$. tongmaiensis PCGs of the mitogenome. 

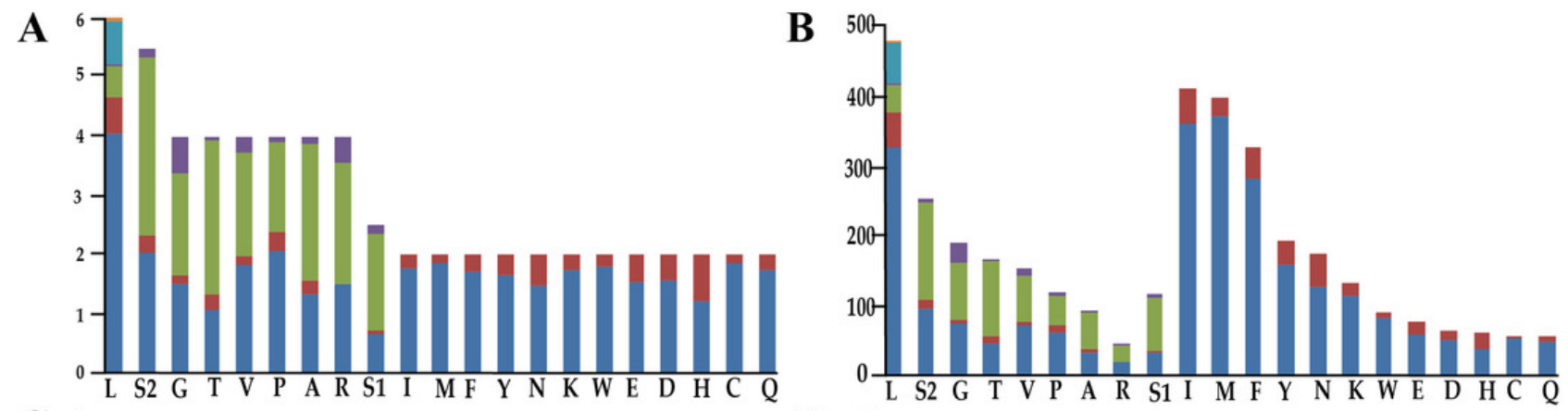

C

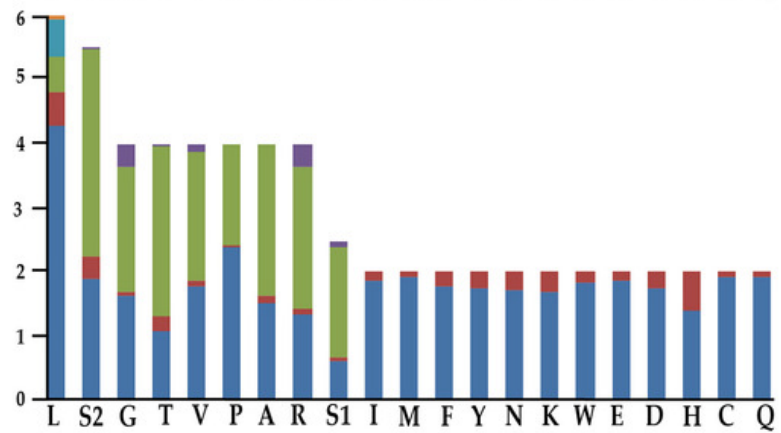

E

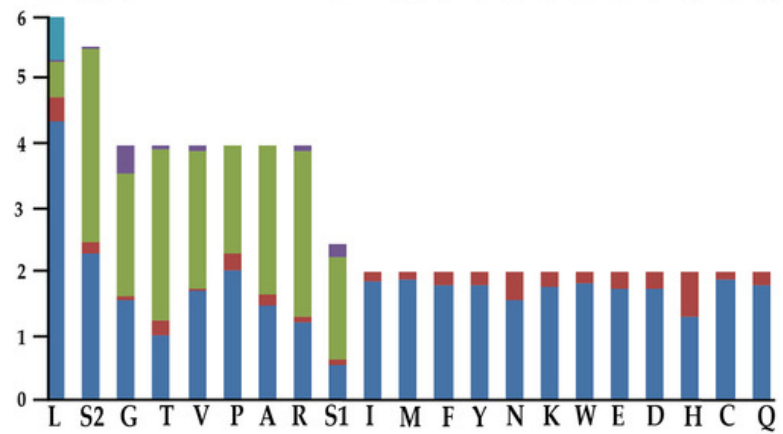

G

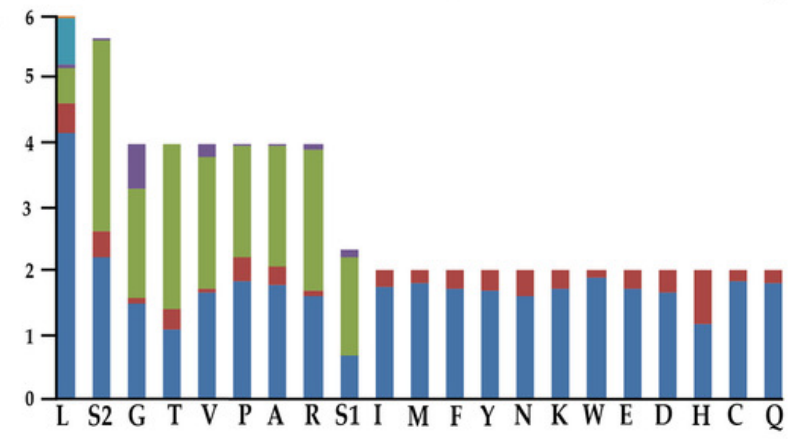

I

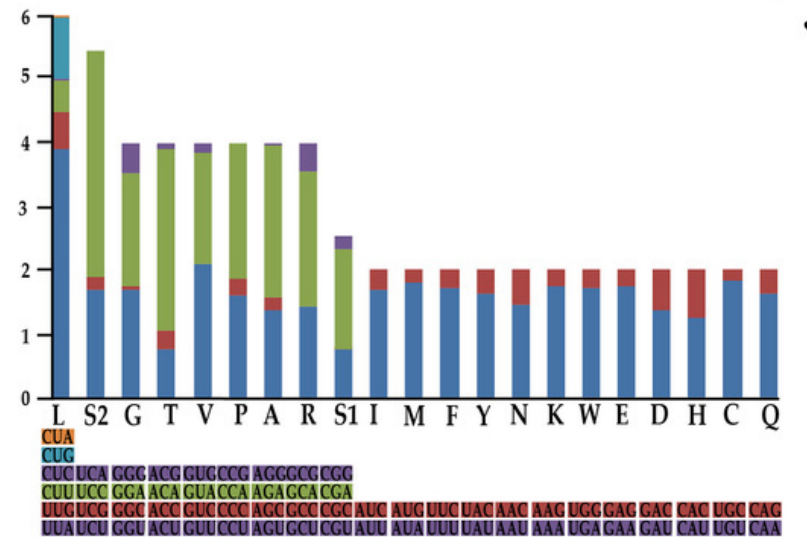

D 500

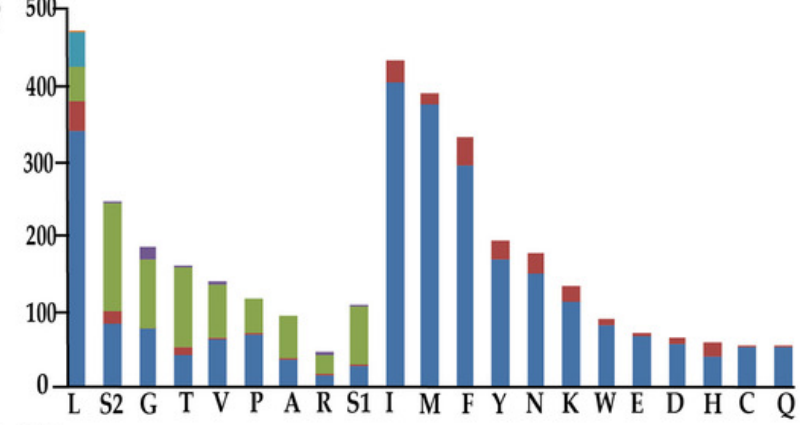

F 500

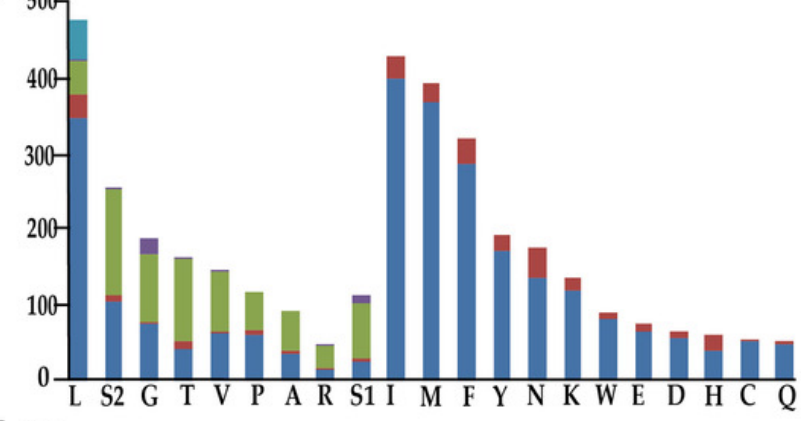

H 50

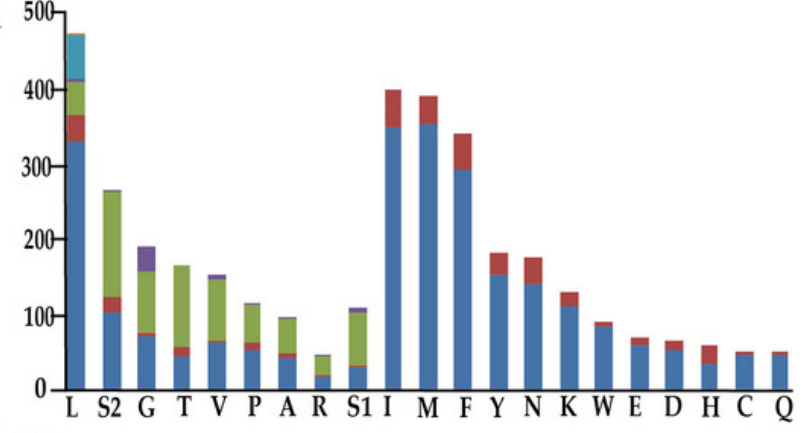

$\mathbf{J}$

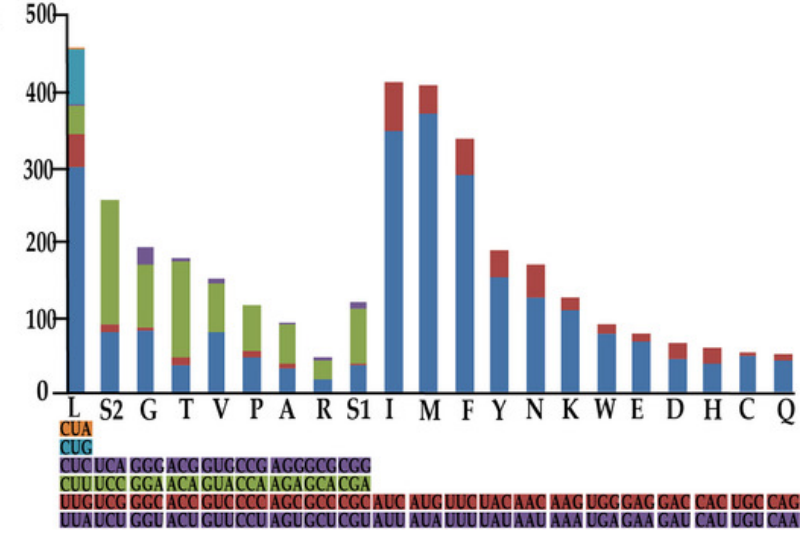




\section{Figure 3}

Figure 3. Predicted secondary structures of tRNAs in of the five Olidiana species.

(A) Predicted secondary structures of Isoleucine (Ile, I). (B) Predicted secondary structures of Glutamine (Glu, Q). (C) Predicted secondary structures of Methioine(Met, M). (D) Predicted secondary structures of Tryptophan (Trp, W). (E) Predicted secondary structures of Cysteine ( Cys, C). (F) Predicted secondary structures of Tyrosine ( Tyr, Y). (G) Predicted secondary structures of Leucine (Leu, L1). (H) Predicted secondary structures of Lysine (Lys, K). (I) Predicted secondary structures of Aspartic (Asp, D). (J) Predicted secondary structures of Glycine (Gly, G). (K) Predicted secondary structures of Tyrosine (Tyr, Y). (L) Predicted secondary structures of Arginine (Arg, R). (M) Predicted secondary structures of Asparagine (Asn, N). (N) Predicted secondary structures of Serine (Ser, S1). (O) Predicted secondary structures of Glutamic (Glu, E). (P) Predicted secondary structures of Phenylalanine (Phe, F). (Q) Predicted secondary structures of Histidine (His, H). (R) Predicted secondary structures of Threonine (Thr, T). (S) Predicted secondary structures of Proline (Pro, P). (T) Predicted secondary structures of Serine (Ser, S2). (U) Predicted secondary structures of Leucine (Leu, L2). (V) Predicted secondary structures of Valine (Val, V). Dashes (-) indicate Watson-Crick base pairing and dots $(\bullet)$ indicate $\mathrm{G}-U$ base pairing. 

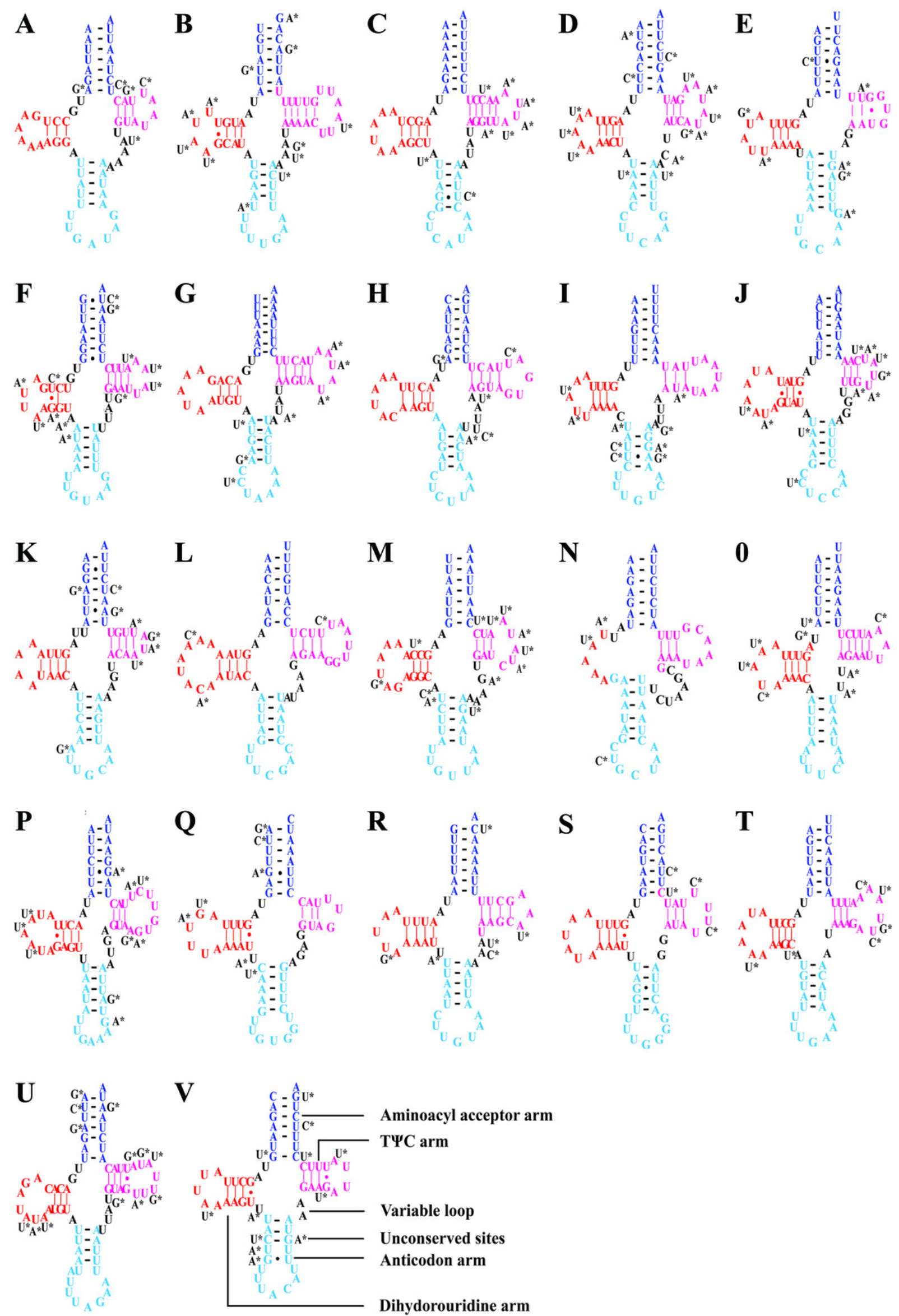

Peer] reviewing PDF | (2020:10:53788:2:0:NEW 10 Feb 2021) 
Figure 4

Figure 4. Organization of the control region in the complete mitogenome of five Olidiana species.

(A) Organization of the control region in the complete mitogenome of Olidiana alata. (B)

Organization of the control region in the complete mitogenome of Olidiana longsticka. (C)

Organization of the control region in the complete mitogenome of Olidiana olbliquea. (D)

Organization of the control region in the complete mitogenome of Olidiana ritcheri. (E)

Organization of the control region in the complete mitogenome of Olidiana tongmaiensis.

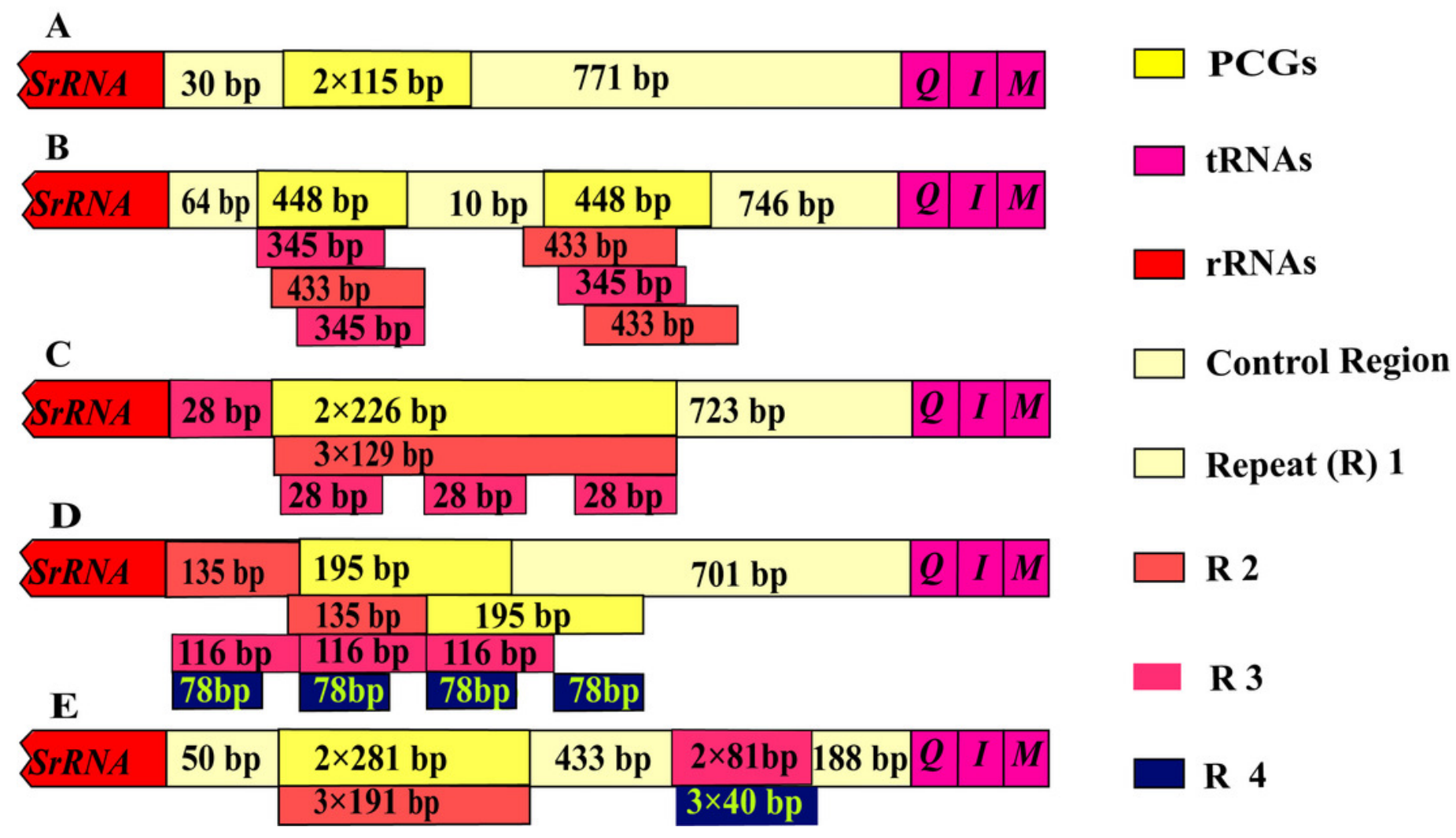


Figure 5

Figure 5. Phylogenetic trees of Cicadellidae inferred by MrBayes 3.2.6 based on nucleotides of the first and second codons of 13 PCGs and 2 rRNAs (BI-13PCG12-2R). 


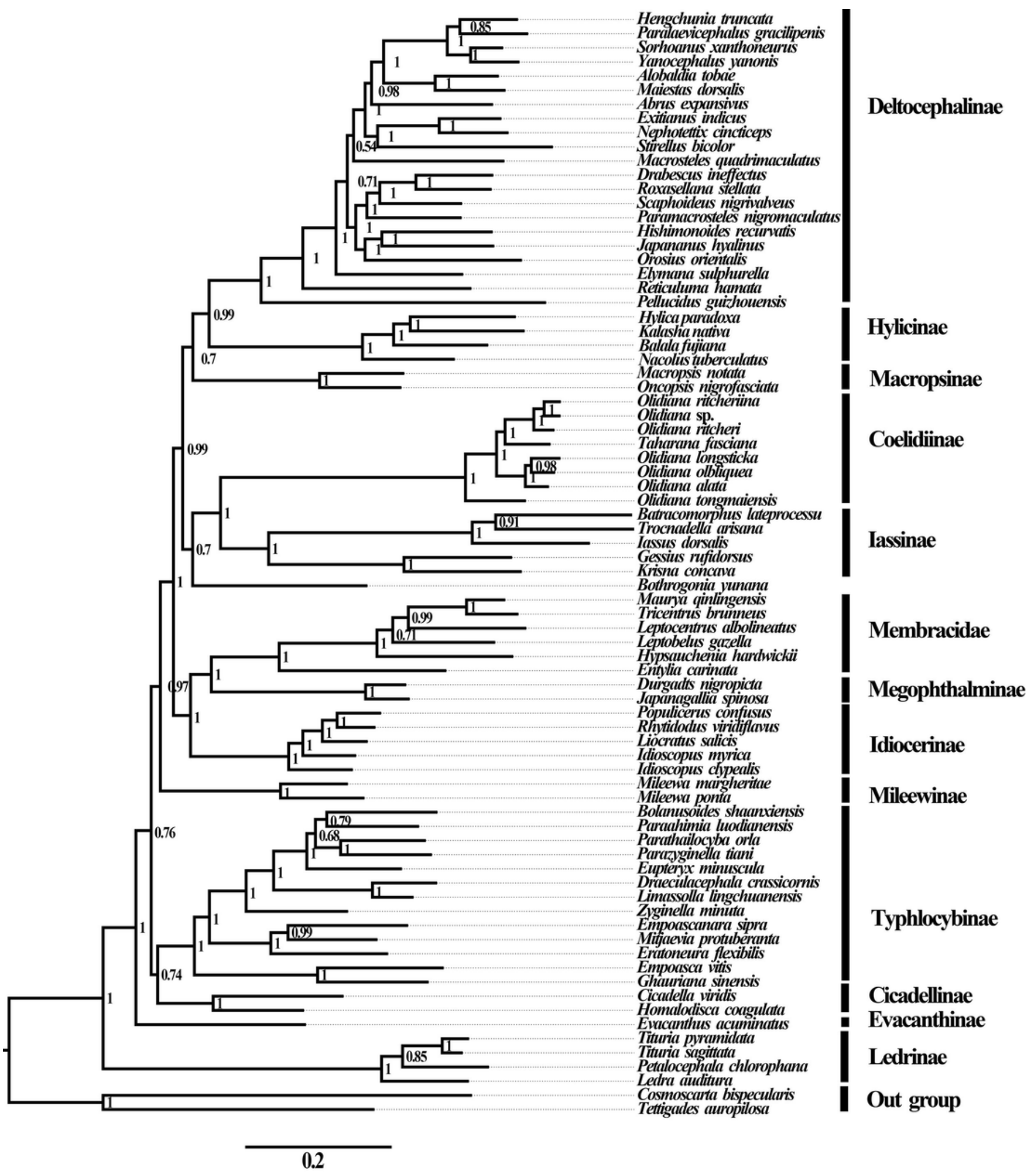




\section{Figure 6}

Figure 6. Phylogenetic trees of leafhoppers.

(A) Phylogenetic trees of leafhoppers inferred by the MrBayes 3.2.6 and maximum likelihood methods (BI-13PCG12/BI-13PCG12-2R/ML-13PCG-2R). (B) Phylogenetic trees of leafhoppers inferred by the maximum likelihood method based on nucleotide sequences from the first and second codons of 13 PCGs (ML-PCG12). (C) Phylogenetic trees of leafhoppers inferred by the MrBayes 3.2.6 based on nucleotides of the first and second codons of 13 PCGs and 2 rRNAs (BI-PCG12-2R); and maximum likelihood 13 PCGs and 2 rRNAs (ML-13PCG-2R). (D) Phylogenetic trees of leafhoppers inferred by the maximum likelihood 13 PCGs (ML-13PCG).

(E) Phylogenetic trees of leafhoppers inferred by the maximum likelihood 13 PCGs and 2 rRNAs (ML-13PCG-2R). (F) Phylogenetic trees of leafhoppers inferred by the maximum likelihood and MrBayes 3.2.6 methods based on amino acid sequences of 13 PCGs (ML/BI$A A)$. 


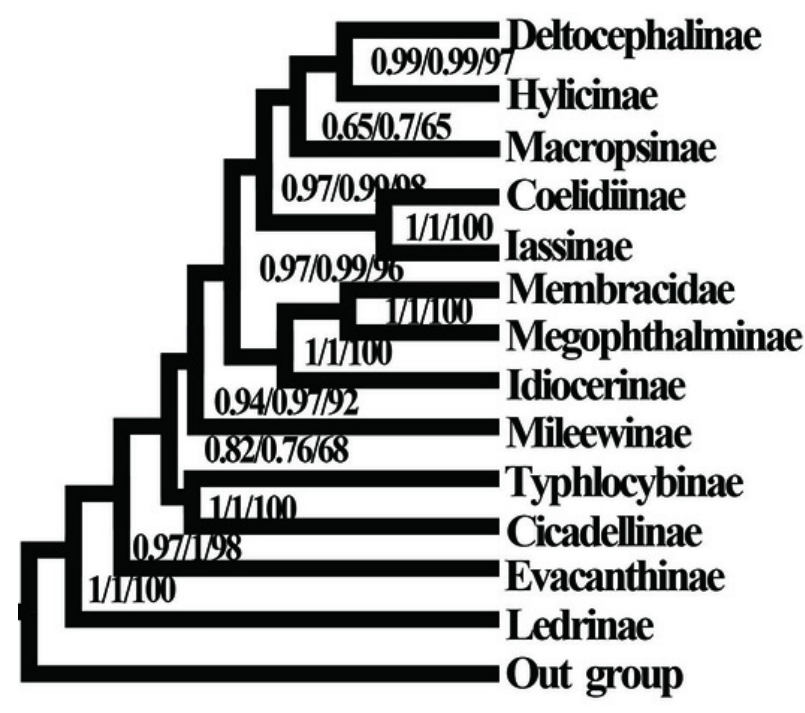

A

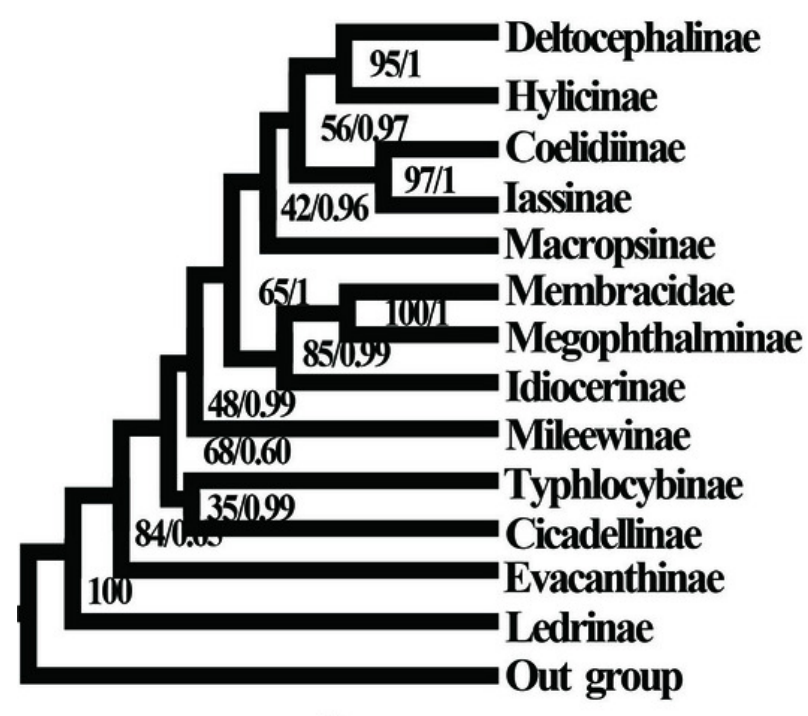

C

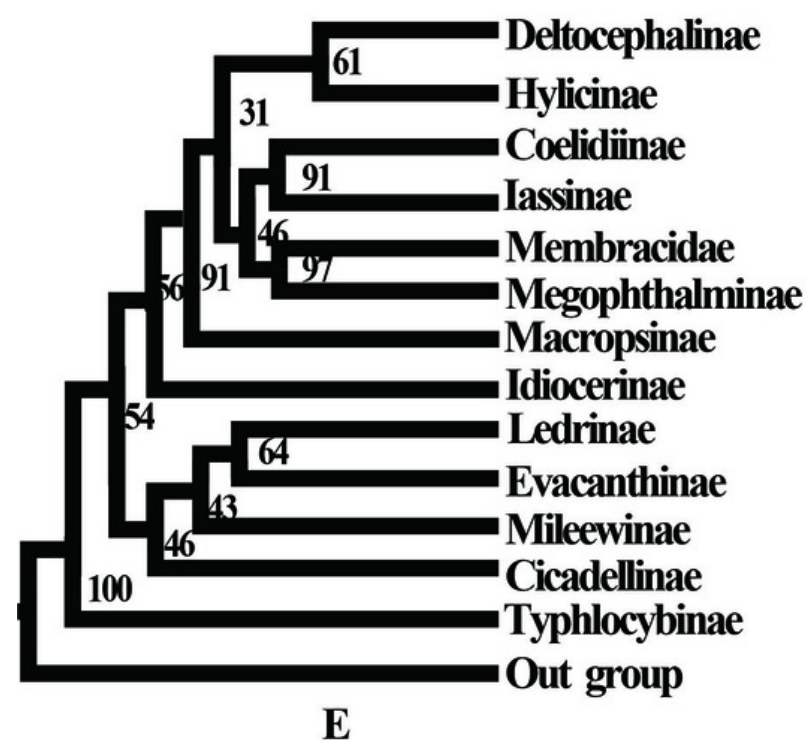

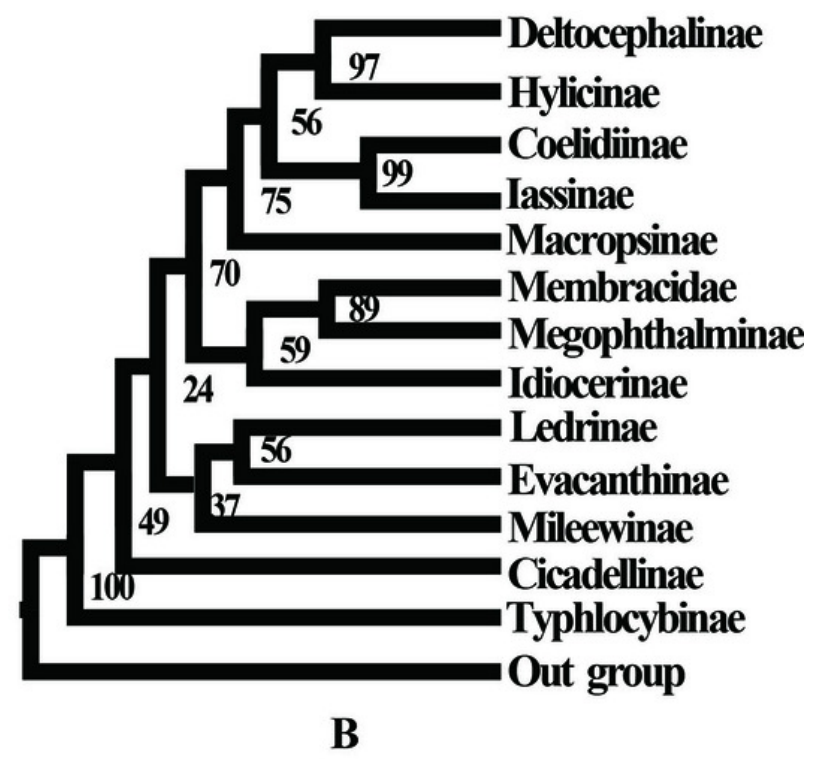

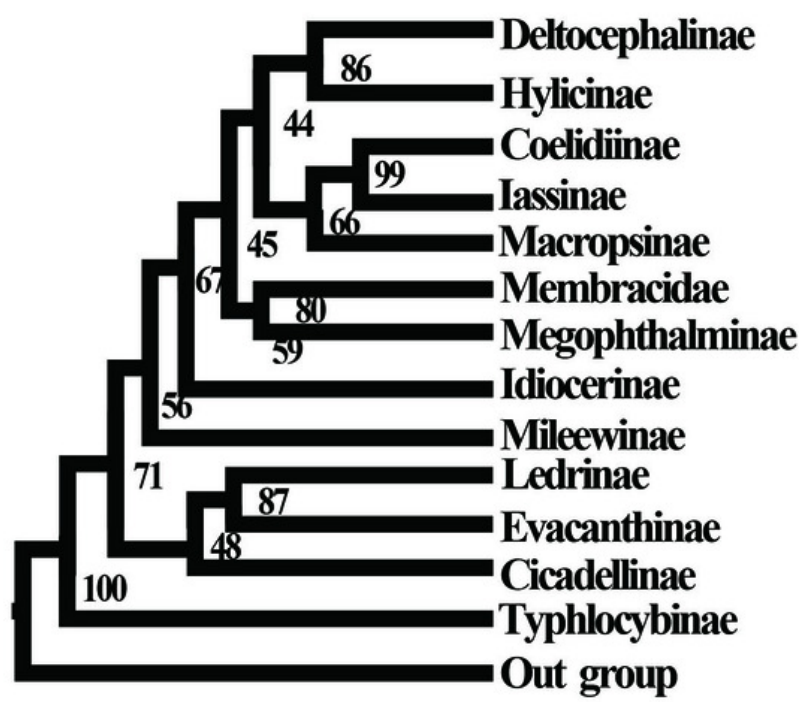

D

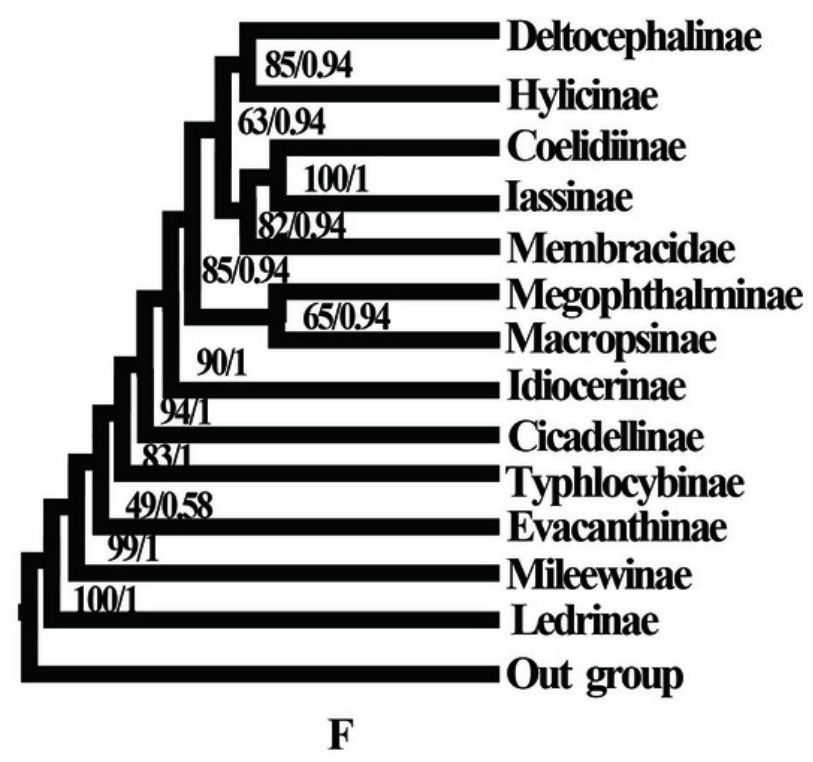




\section{Table $\mathbf{1}$ (on next page)}

Table 1. Nucleotide composition and skewness of five Olidiana mitogenomes. 


\begin{tabular}{|c|c|c|c|c|c|c|}
\hline Regions & Species & Length (bp) & $\operatorname{AT}(\%)$ & GC $(\%)$ & AT skew & GC skew \\
\hline \multirow[t]{5}{*}{ Whole genome } & O. alata & 15,205 & 78.0 & 22.0 & 0.169 & -0.245 \\
\hline & O. longsticka & 15,993 & 79.7 & 20.3 & 0.147 & -0.202 \\
\hline & O. olbliquea & 15,312 & 79.3 & 20.7 & 0.155 & -0.227 \\
\hline & O. ritcheri & 15,372 & 78.2 & 21.8 & 0.151 & -0.257 \\
\hline & O. tongmaiensis & 15,363 & 78.1 & 21.9 & 0.195 & -0.269 \\
\hline \multirow[t]{5}{*}{22 tRNAs } & O. alata & 1,411 & 79.4 & 20.6 & 0.118 & -0.126 \\
\hline & O. longsticka & 1,410 & 79.3 & 20.7 & 0.111 & -0.130 \\
\hline & O. olbliquea & 1,400 & 79.6 & 20.4 & 0.111 & -0.110 \\
\hline & O. ritcheri & 1,400 & 78.9 & 21.1 & 0.118 & -0.147 \\
\hline & O. tongmaiensis & 1,411 & 79.3 & 20.7 & 0.145 & -0.150 \\
\hline \multirow[t]{5}{*}{13 PCGs } & O. alata & 10,998 & 78.5 & 23.3 & 0.157 & -0.253 \\
\hline & O. longsticka & 10,890 & 76.6 & 21.4 & 0.158 & -0.234 \\
\hline & O. olbliquea & 10,890 & 78.2 & 21.8 & 0.166 & -0.239 \\
\hline & O. ritcheri & 10,887 & 77.0 & 23.0 & 0.169 & -0.261 \\
\hline & O. tongmaiensis & 10,886 & 76.6 & 23.4 & 0.214 & -0.299 \\
\hline \multirow[t]{3}{*}{2 rRNAs } & O. alata & 1,915 & 80.7 & 19.3 & 0.199 & -0.306 \\
\hline & O. longsticka & 1,915 & 82.1 & 17.9 & 0.189 & -0.307 \\
\hline & O. olbliquea & 1,968 & 81.4 & 18.6 & 0.199 & -0.290 \\
\hline
\end{tabular}




\begin{tabular}{|c|c|c|c|c|c|c|}
\hline & O. ritcheri & 1,955 & 81.3 & 18.7 & 0.171 & -0.294 \\
\hline & O. tongmaiensis & 1,911 & 81.0 & 19.0 & 0.231 & -0.284 \\
\hline \multirow[t]{5}{*}{ Control region } & O. alata & 1,017 & 85.3 & 14.7 & 0.048 & -0.061 \\
\hline & O. longsticka & 1,804 & 84.1 & 15.9 & 0.065 & 0.057 \\
\hline & O. olbliquea & 1,075 & 85.8 & 14.2 & 0.042 & -0.085 \\
\hline & O. ritcheri & 1,149 & 84.4 & 15.6 & 0.012 & -0.231 \\
\hline & O.tongmaiensis & 1,164 & 85.3 & 14.7 & 0.048 & -0.061 \\
\hline
\end{tabular}

1

2 